\title{
Specificity for Reach Planning in the Human PPC
}

\author{
Marco Ciavarro ${ }^{1,2,3}$ and Ettore Ambrosini ${ }^{2,3}$ \\ ${ }^{1}$ Department of Human and General Physiology, University of Bologna, 40126 Bologna, Italy, ${ }^{2}$ Laboratory of Neuropsychology and Cognitive Neuroscience, \\ Department of Neuroscience and Imaging, University G. d'Annunzio, 66013 Chieti-Pescara, Italy, and ${ }^{3}$ Institute for Advanced Biomedical Technologies, \\ Foundation University G. d'Annunzio, 66013 Chieti, Italy \\ Review of Vesia et al.
}

Research in both humans and macaques has provided evidence that the posterior parietal cortex (PPC) serves as a sensorymotor interface crucially involved in visuomotor transformations for goaldirected movements. It is well accepted that in macaque monkeys, these transformations manifest themselves as a gradual change in the functional organization along gradients of effector preferences. For example, several single-unit recording studies have demonstrated that different cortical areas within the PPC represent plans for eye and arm movements (Snyder et al., 2000). Planning eye movements involves a functional class of neurons on the lateral bank of the intraparietal sulcus (LIP area) (Colby et al., 1996), whereas reach-related activity has been described along the dorsomedial visual stream in the superior parietal lobule, including area 5 [an area located in the middle portion of the intraparietal sulcus (MIP)] and a more posterior region in the medial superior parieto-occipital cortex (V6A) (Galletti et al., 2003). In humans, functional imaging studies reported a less clear segregation between reach- and saccaderelated areas and the debate on effector specificity in human PPC is still open (Levy et al., 2007; for review, see Filimon, 2010).

Received Nov. 3, 2010; revised Dec. 7, 2010; accepted Dec. 10, 2010. Correspondence should be addressed to Ettore Ambrosini, Department of Neuroscience and Imaging, University of Chieti, Via dei Vestini 33, 66013 Chieti, Italy. E-mail: e.ambrosini@unich.it.

DOI:10.1523/JNEUROSCI.5772-10.2011

Copyright $\odot 2011$ the authors $\quad 0270-6474 / 11 / 312719-02 \$ 15.00 / 0$
In a recent study published in The Journal of Neuroscience, Vesia et al. (2010) used transcranial magnetic stimulation (TMS) to determine effector specificity in human PPC. This technique allows one to interfere with a specific stage of visuomotor transformation in a particular cortical area. Vesia et al. (2010) used online $10 \mathrm{~Hz}$ repetitive TMS (rTMS) to examine the specific functional role of one posteriormedial site [superior parieto-occipital cortex (SPOC), which likely includes area V6A] and two anterior-lateral parietal sites [angular gyrus (AG) and midposterior intraparietal sulcus (mIPS), which likely includes LIP and MIP areas] in the planning of saccades versus reaches. Vesia et al. (2010) conducted three separate experiments using a delayed saccade and reach paradigm with six visual targets (aligned horizontally in steps of $10^{\circ}$ from $30^{\circ}$ left to $30^{\circ}$ right). In the first experiment, subjects were required to perform saccades or reaching movements (randomly interleaved) with the right hand in complete darkness; in the second experiment, reaching movements were performed with the left hand (again in complete darkness); and in the last one, reaching movements were performed with the right hand, but now with visual feedback (Vesia et al., 2010, their Fig. 3). Task performance was evaluated in terms of movement accuracy and precision. Accuracy was measured as the signed difference between mean endpoint and target positions (constant errors), whereas precision was measured as the area of $95 \%$ confidence ellipses fitted to endpoint distributions (variable errors).

One of the main findings of this study was the reduced accuracy of saccades and the reduced precision of reaches to contralateral targets after stimulation over right mIPS and AG. The TMS-induced effect in these anterior-lateral sites was further reduced by the visual feedback of the hand and, more importantly, was limbspecific, showing a contralateral limbrelated bias in precision measures. In contrast, stimulation of SPOC in either hemisphere did not affect saccade precision or accuracy and did produce a significant shift of mean horizontal reach endpoints toward central fixation (i.e., hypometria), which persisted even when the hand was visible. Based on these data, Vesia et al. (2010) propose that SPOC is specialized for encoding reach targets in retinal coordinates, whereas the more anterior-lateral parietal regions are involved in computations for both reach and saccade motor vectors. Moreover, the authors interpret their results to suggest a caudorostral gradient in limb selectivity (caudally absent and rostrally present).

It is worth noting, however, that limb specificity was assessed by considering only the reach precision measure, without taking into account what emerged from the analysis on reach accuracy. Whereas reach precision clearly revealed contralateral limb specificity of the two anteriorlateral areas (Vesia et al., 2010, their Fig. 7), reach accuracy (Vesia et al., 2010, their Fig. 6, $D-G$ ) showed a significantly greater 
hypometria following rTMS over right SPOC during right hand reaches (experiments 1 and 3) and following rTMS over left SPOC during left hand reaches (experiment 2). This suggests the potential presence of a TMS-induced limb-specific effect for the SPOC area also. Unfortunately, this result was not discussed in Vesia et al.'s (2010) section on limb specificity. An ipsilateral limb specificity in SPOC would be inconsistent with most monkey and neuropsychological evidence (Karnath and Perenin, 2005; Chang et al., 2008; but see Busan et al., 2009). Moreover, it would weaken the conclusion drawn by the authors about the involvement of SPOC in reach planning. In fact, if the stimulation over SPOC induces some effects modulated not only by target eccentricity, but also by the reaching hand, then it is unlikely that SPOC function is restricted to encoding reach goals in retinal coordinates. This function should indeed be independent from nonretinal factors, such as handedness.

From reach accuracy results it is possible to observe another interesting trend, namely the potential presence of an effect on horizontal errors resulting from the stimulation of the two anterior-lateral sites. Indeed, rTMS over right MIPS and AG seems to induce horizontal hypometria in the contralateral visual hemifield, but only for reach movements performed with the right hand (Vesia et al., 2010, their Figs. 6 and S1, $H-I$ ). Although reach accuracy was significantly affected only by stimulation over SPOC, the trend reported above would be in agreement with previous work (van Donkelaar and Adams, 2005) and would bring further support to the involvement of anterior-lateral regions in reach planning.

Another aspect of the results that deserves attention concerns the influence of visual hemifield and target eccentricity on reach accuracy. Stimulation over left SPOC produced a significant horizontal hypometria for left hand reaches only in the right visual hemifield (Vesia et al., 2010, their Fig. 6D). Furthermore, it can be noted that, for right-hand reaches, the greater deviation of end points toward central fixation in the visual hemifield contralateral to the stimulated site seems to be present for both right and left SPOC (Vesia et al., 2010, their Fig. 6G). Unfortunately, pairwise comparisons concerning TMS-induced effects between the two SPOC have not been provided, thus preventing further speculation on this point. Moreover, reach accuracy results showed a significant effect of stimulation over SPOC only for the two peripheral targets, consistent with neuropsychological and functional findings that suggest specificity of this region for reaching in peripheral vision (Karnath and Perenin, 2005; Prado et al., 2005). Therefore, to appraise more specifically the influence of visual hemifield and target eccentricity, it would be helpful in future experiments to include them in the factorial design, i.e., splitting the six levels of target location factor in a 2 (visual hemifield) $\times 3$ (target eccentricity) factorial design.

In summary, in the debate on saccade and reach specificity in human PPC, Vesia et al. (2010) have used an innovative method (rTMS) that allows establishing a causal link between the function of a particular cortical region and behavioral performance. Using this approach, which is complementary to functional neuroimaging, Vesia et al. (2010) have provided evidence for effector specificity in human PPC, showing a caudorostral gradient of effector preferences. Extending previous results (Hagler et al., 2007), they suggest that anterior-lateral parietal areas mIPS and $A G$ are involved in the encoding of both saccade and reach movements. Moreover, this work identified the clear specificity of SPOC in the planning of reaches only, although further studies are needed to clarify the role of both visual hemifield and limb specificity on these complex visuomotor transformations.

\section{References}

Busan P, Jarmolowska J, Semenic M, Monti F, Pelamatti G, Pizzolato G, Battaglini PP (2009) Involvement of ipsilateral parietooccipital cortex in the planning of reaching movements: evidence by TMS. Neurosci Lett 460:112-116.

Chang SW, Dickinson AR, Snyder LH (2008) Limb-specific representation for reaching in the posterior parietal cortex. J Neurosci 28 : $6128-6140$.

Colby CL, Duhamel JR, Goldberg ME (1996) Visual, presaccadic, and cognitive activation of single neurons in monkey lateral intraparietal area. J Neurophysiol 76:2841-2852.

Filimon F (2010) Human cortical control of hand movements: parietofrontal networks for reaching, grasping, and pointing. Neuroscientist 16:388-407.

Galletti C, Kutz DF, Gamberini M, Breveglieri R, Fattori P (2003) Role of the medial parietooccipital cortex in the control of reaching and grasping movements. Exp Brain Res 153: $158-170$.

Hagler DJ Jr, Riecke L, Sereno MI (2007) Parietal and superior frontal visuospatial maps activated by pointing and saccades. Neuroimage 35:1562-1577.

Karnath HO, Perenin MT (2005) Cortical control of visually guided reaching: evidence from patients with optic ataxia. Cereb Cortex 15:1561-1569.

Levy I, Schluppeck D, Heeger DJ, Glimcher PW (2007) Specificity of human cortical areas for reaches and saccades. J Neurosci 27:4687-4696.

Prado J, Clavagnier S, Otzenberger H, Scheiber C, Kennedy H, Perenin MT (2005) Two cortical systems for reaching in central and peripheral vision. Neuron 48:849-858.

Snyder LH, Batista AP, Andersen RA (2000) Intention-related activity in the posterior parietal cortex: a review. Vis Res 40:1433-1441.

van Donkelaar P, Adams J (2005) Gaze-dependent deviation in pointing induced by transcranial magnetic stimulation over the human posterior parietal cortex. J Mot Behav 37:157-163.

Vesia M, Prime SL, Yan X, Sergio LE, Crawford JD (2010) Specificity of human parietal saccade and reach regions during transcranial magnetic stimulation. J Neurosci 30:13053-13065. 